\title{
Effectiveness of Inversion Diversity to Cope with EMI within a Two-Channel Redundant System
}

\author{
Jonas Lannoo, Andy Degraeve, Dries Vanoost, Jeroen Boydens and Davy Pissoort \\ M-Group (Mechatronics Group) \\ KU Leuven, Bruges Campus \\ Spoorwegstraat 12, B-8200 Bruges, Belgium \\ jonas.lannoo@kuleuven.be, andy.degraeve@kuleuven.be, dries.vanoost@kuleuven.be, \\ jeroen.boydens@kuleuven.be, davy.pissoort@kuleuven.be
}

\begin{abstract}
This paper studies the effectiveness of inversion diversity to cope with EM disturbances caused by strong incident fields under reverberation room conditions. Here, reverberation room conditions refers to the situation where multiple plane waves are incident onto the system-under-study, each with a random angle-of-incidence, polarization and phase. Two different geometries are compared: a non-redundant system comprising a single trace on a PCB and a redundant system comprising two parallel traces on a single $\mathrm{PCB}$. A reciprocity-based technique is used to efficiently calculate the induced voltages and the resulting bit error probability in the different geometries. It is shown that with increasing distance between the two traces, the probability on false positive (i.e. two identical, but incorrect bits at the comparator) increases.
\end{abstract}

Keywords-EMI Risk Management, electromagnetic diversity, redundancy, functional safety

\section{INTRODUCTION}

Electromagnetic interference (EMI), familiar to most people as the buzzing sound from a loudspeaker when a mobile-phone call is received, is becoming much more than just an annoyance. A lot of the high-tech electronic equipment in our cars, homes, workplaces and hospitals is increasingly vulnerable to EMI. Over the coming years, we will witness without doubt an increasing amount of semi- or fully-autonomous systems in our day-to-day life. Examples include, amongst others, autonomous vehicles, autonomous ships, unmanned aerial vehicles, drones, smart machines, smart production environments, and robot care systems. Common to all the above examples is that programmable electronics is key to their functioning and that programmable electronics is used to fulfil mission- or safety-critical specifications. Unfortunately, all electronic devices are increasingly vulnerable to Electro-Magnetic Interference (EMI). For these reasons, combined expertise in Electro-Magnetic Compatibility (EMC) Engineering and Functional Safety Engineering will gain huge importance within many industrial sectors of vital importance to our society and economy.

Unfortunately, EMC engineering shares very few concepts and terminology with Functional Safety, as EMC engineering is driven by showing compliance to legislative rules like the EMC directive (2014/30/EU) [1]. As a result, EMC engineering currently follows a rule- and test-based approach. During design a number of guidelines are prescribed which result in the default application of a set of mitigation techniques (filtering, shielding, cable routing etc.). Testing is then used to establish whether the system meets the requirements of EMC standards or not. However, this rule-based approach has several significant shortcomings. Recent literature [2]-[5] has shown that classical EMI shielding, grounding, filtering and testing on their own can never lead to a feasible and economically affordable approach to guarantee safety due to the many possible variations of EMI that can occur during the lifetime of an electronic system. As is described in the most recent IET Guide on EMC for Functional Safety [6] as well as in the IEEE Standard draft P1848 [7] on Techniques \& Measures to Manage Functional Safety and Other Risks With Regard to Electromagnetic Disturbances, a totally new methodology imposes itself where the classical EMI mitigation techniques are complemented with appropriate hardening Techniques and Measures (T\&Ms) which are typical for Functional Safety. Unfortunately, none of these T\&Ms have been originally developed to deal with EMI. So, they need to be improved, adapted and their effectiveness for EMI needs to be demonstrated.

One such hardening technique is the use of redundancy and voting. This means that one implements two or more identical sets of hardware and software receiving the same inputs and performing the same operations. When a malfunction occurs in one of these 'parallel channels', a comparator detects that their outputs no longer agree and triggers appropriate actions to maintain safety. Unfortunately, EMI is a complex phenomenon which has to be seen as a systematic, common cause failure. Indeed, "systematic" because a given system design will always behave in the same way when a given EMI is applied. "Common-cause" because EMI influences many different components at the same time. As EMI is a systematic common-cause failure, the malfunctions that EMI creates in parallel channels can easily be so similar that the comparator cannot detect that there is a problem at all. An important challenge is how to apply, in a cost-effective way, EMI-diverse parallel channels within a redundant system. With EMI-diverse, we mean here a system where the output response is different for the same incident EMI.

In [8]-[10], the effectiveness of spatial diversity with a 2-out-of3 (2003) redundant system was studied in case of electromagnetic disturbance comprises strong incident fields under single plane wave or reverberation room conditions. Here, reverberation room conditions refers to the situation where multiple plane waves are incident onto the system-under-study, each with a random angle-of-incidence, polarization and phase. In [11], another 
possibility to obtain EMI-diverse parallel channels is studied, namely the use of different termination schemes within the parallel channels: source-termination, load-termination and sourceand-load termination. This diversity technique is studied under reverberation room conditions, as that is the most representative for real-world situations.

In this paper, the focus is on the use of inversion within a 2channel redundant system. Note that there is a significant difference with respect to what can be obtained with a 2-channel redundant system compared to a 2003 system. Indeed, with a 2003 system one can use majority voting to obtain fault-tolerant behavior. In contrast, with a 2-channel system, one can only detect that an error has occurred (when both channels have a different outcome) upon which one can trigger and appropriate action (e.g. transition to a fail-safe state). However, what needs to be avoided at all times is that both outcomes give the same, but wrong output, so-called false positives.

The remainder of this paper is organized as follows. Section II describes the geometry under study. Section III briefly explains the reciprocity-based technique to efficiently calculate the induced voltages with the 2-channel system. Section IV details how to obtain the resulting bit error probability. Section V numerically compares the effectiveness of inversion to cope with EMI. Section VI draws concluding remarks and gives directions for future work.

\section{GEOMETRY UNDER STUDY}

Two different geometries will be compared:

1. A single Printed Circuit Board (PCB) with a single 50 Ohm microstrip (Fig. 1). This is the reference situation (non-redundant system). In this case, the microstrip is driven by a $1 \mathrm{~V}$ source with a $50 \mathrm{Ohm}$ output impedance while at its other end it is connected to a $50 \mathrm{Ohm}$ load.

2. A single PCB with two parallel, identical $50 \mathrm{Ohm} \mathrm{mi-}$ crostrips (Fig. 2). All microstrips are driven from $1 \mathrm{~V}$ sources with $50 \mathrm{Ohm}$ output impedance and connect to $50 \mathrm{Ohm}$ loads. On one of the traces, the signal will be inverted. Several variants of this configuration will be considered which differ in the distance between the two traces (measured from center to center).

In all cases, the PCB is a FR4 substrate of $10 \mathrm{~cm}$ by $16 \mathrm{~cm}$ and $1.6 \mathrm{~mm}$ thickness. All microstrips have a width of $3 \mathrm{~mm}$, a length of $5 \mathrm{~cm}$ and a variable distance between them.

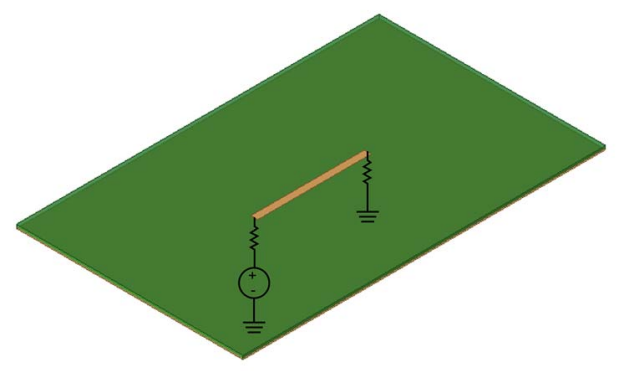

Fig. 1. Single $\mathrm{PCB}$ with one $50 \mathrm{Ohm}$ microstrip (reference situation)

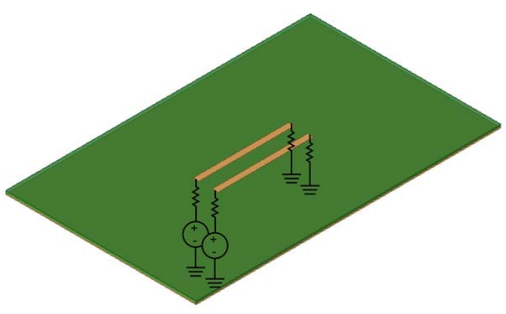

Fig. 2. Single PCB with two $50 \mathrm{Ohm}$ microstrip (2-channel system). Various distances between the microstrips are considered.

\section{EFFICIENT CALCULATION OF THE INDUCED VOLTAGES UNDER REVERBERATION ROOM CONDITIONS}

In order to efficiently calculate the induced voltages at the ends of the microstrips under reverberation room conditions, the antenna-reciprocity based algorithm proposed in [12] is used. For predicting the coupling of plane waves to a system-undertest, the plane-wave representation of a realistic EM environment with many reflections is used [13][14]. In [13][14], the fields within a reverberation room are represented in a statistical means by using the plane-wave integral representation. This representation states that the fields within a reverberation chamber can be described by superposing a large number of independent plane waves coming from arbitrary directions and with arbitrarily varying polarizations and phases. As a result the resulting induced voltages and currents can be obtained by a Monte Carlo simulation in which one considers $M$ experiments each of which comprising $N$ plane waves.

A brute-force method where a full wave simulation is done for every separate plane-wave, would lead to exponential simulation times. In contrast, the methodology used in this paper is very efficient as it requires only one full-wave simulation per port within the system-under-study. Briefly described, this methodology comprises three main steps:

1. Run a full-wave simulation of the geometry-understudy as a radiating multi-port antenna system.

2. Calculate the system's impedance matrix and far-field patterns;

3. Use the antenna-reciprocity theorem to calculate the induced voltage or current for an arbitrary incoming plane wave.

As a result, only a limited number of full-wave simulations (i.e. one per port) is needed. The reader is referred to [12] for full details on this reciprocity-based method.
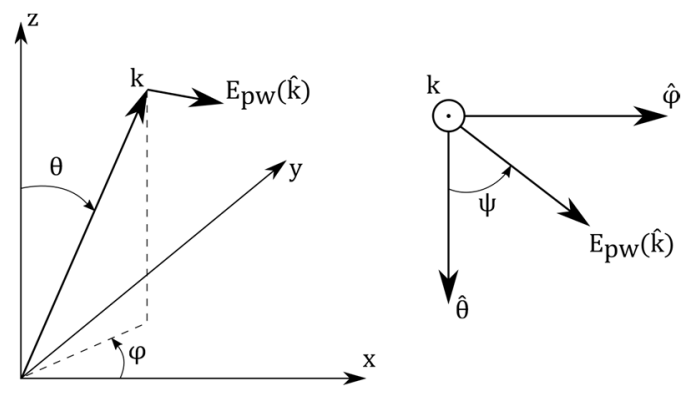

Fig. 3. Configuration of the different angles of incidence and polarization. 
In the statistical plane-wave representation of a reverberation room like environment, care has to be taken to correctly represent the statistical behavior of the incoming plane waves. No direction of arrival, no polarization or no phase should get any privilege. In this paper, we use the reasoning described in [13] for the statistical distribution of the different parameters (spherical coordinates) (Fig. 3):

- The azimuth angle $\varphi$ is uniformly distributed in the range $[0,2 \pi]$.

- The polar angle $\theta$ is chosen such that $\cos (\theta)$ is uniformly distributed in the range $[-1,1]$.

- The polarization angle $\psi$ is uniformly distributed in the range $[0, \pi]$.

- The phase angle $\alpha$ is uniformly distributed in the range $[0,2 \pi]$.

In order to obtain the correct (average) field strength after superposing all random plane waves, the amplitude of each separate plane wave is normalized by $1 / \sqrt{N}$.

In what follows, $M$ and $N$ are chosen to be 10.000 and 200, respectively for the Monte Carlo Simulation.

\section{CALCULATION OF THE BIT ERROR RATE}

In this paper, the Bit Error Rate (BER) is calculated by disturbing a bit pattern of 100 randomly chosen bits (50/50 probability for a ' 0 ' and a ' 1 ') - transmitted over the microstrip(s) with the $M$ different sets of $N$ plane waves mentioned in Section III. The BER is defined as:

$$
\mathrm{BER}=\frac{\text { number of bits received incorrectly }}{M \times \text { length bit pattern }}
$$

The system's impedance matrix, calculated in the first step of the reciprocity-based algorithm described in Section III, can be used to calculate for a given bit pattern (encoded into actual voltage levels) at the input ports of the microstrips, the resulting bit patterns (again expressed in voltage levels) at the output ports of the microstrips.

For the encoding and decoding, the unipolar NRZ-L encoding metrics were used. For encoding, the voltage level for a ' 0 ' and a ' 1 ' at an input port is $0 \mathrm{~V}$ and $1 \mathrm{~V}$, respectively. This results in a theoretical output voltage at the load side of $0.5 \mathrm{~V}$ because the whole system is correctly terminated. For the decoding, the thresholds used to differentiate between a '0' or a ' 1 ' are set to $1 / 3^{\text {th }}$ and $2 / 3^{\text {th }}$ of the theoretical output voltage, respectively. If the voltage is below the $1 / 3^{\text {th }}$ threshold, then a ' 0 ' is seen. The same goes when the voltage is above the $2 / 3^{\text {th }}$ threshold, at which moment a '1' will be read. For any values in between the thresholds, the bit is seen as faulty, to create a worst-case result. For a more easy processing, a decoding technique is used where we don't look at the transient behavior of the received bit patterns, but only at the middle of each received bit. So our analysis does not take in account e.g. any Schmitt-trigger detection technique.

As the focus of this paper is to study the effectiveness of inversion, the bit pattern on one of the two microstrips is inverted. This means that a ' 1 ' is actually sent as a ' 0 ' and vice-versa. So, if on the one trace '101010' would be sent, '010101' would be sent on the other. Before the comparator, the inverted bit pattern is inverted once more, after which the comparator can compare both bit patterns bit-per-bit.
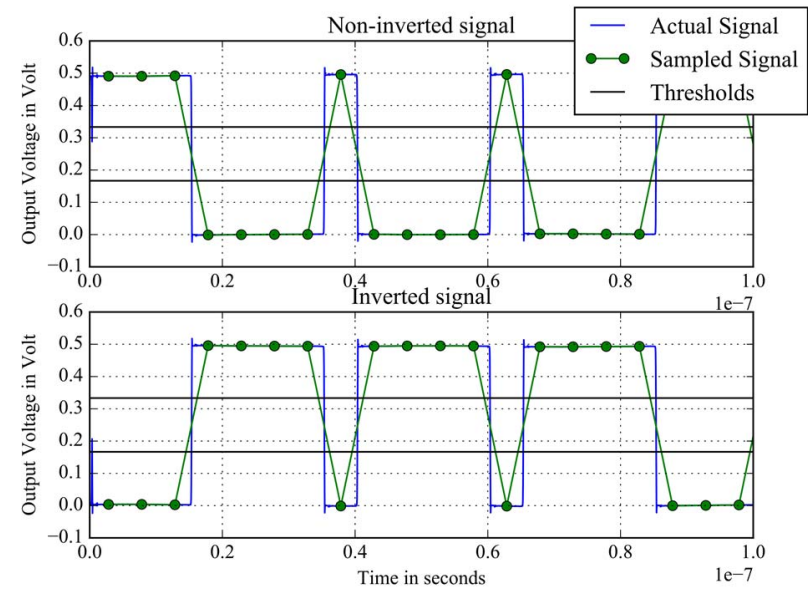

Fig. 4. The non-disturbed digital signals at the outputs of the two traces.

\section{NUMERICAL RESULTS}

In all examples below, the required full-wave simulation of the geometries-under-study were performed with the FDTD solver of EMPro from Keysight Technologies [15]. The reciprocity-based post-processing and BER calculations are done using an in-house developed simulation framework in which the bit pattern, bit pattern frequency, encoding and decoding technique, EMI frequency, etc. can be freely chosen.

For the results given below, the frequency of the EMI disturbance has been fixed to $500 \mathrm{MHz}$, while the frequency of the bit pattern frequency was fixed to $200 \mathrm{MHz}$.
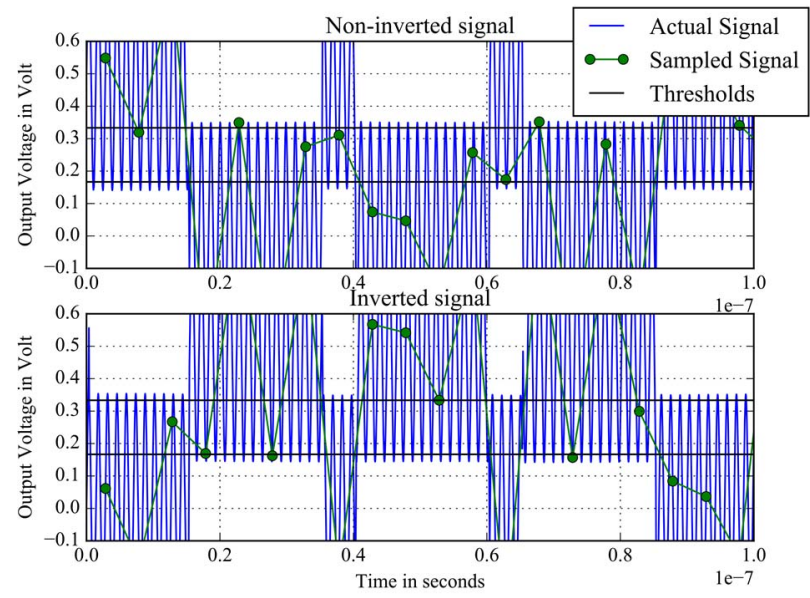

Fig. 5. The disturbed signals at the outputs of the two traces.

In Fig. 4. the two digital signals that propagate over the microstrips can be seen. As mentioned above, one of the signals is inverted, but the same coding and decoding is performed.

Upon disturbance due to EMI (Fig. 5), three different situations can occur at the comparator: 
1. The comparator sees two identical bits which also have the correct value, i.e. the value that was originally sent by the source.

2. The comparator sees two different bits. The comparator can only conclude that something went wrong and trigger an appropriate action, e.g. a transition to a fail-safe state. As there are only two values, the comparator cannot deduce which of the two would be the correct one.

3. The comparator sees two identical bits, but those don't have the correct value, i.e. they are both wrong. The comparator cannot distinguish this situation from situation 2 and will not take any action. This situation is a "false- positive" and should be avoided.

Such a "two faults"-situation of "false-positive" can happen when the induced voltages on the two microstrips have a phase difference that is sufficiently large. In that case, the induced voltage can cause both decoded bits to be wrong. Another possibility is that the induced voltages levels fall between the thresholds, so that the decoder also detects a faulty bit (a voltage level between both thresholds is always considered faulty in this paper). The probability that this happens becomes greater with increasing distance between the traces. Figs. 6 to 8 illustrate this.

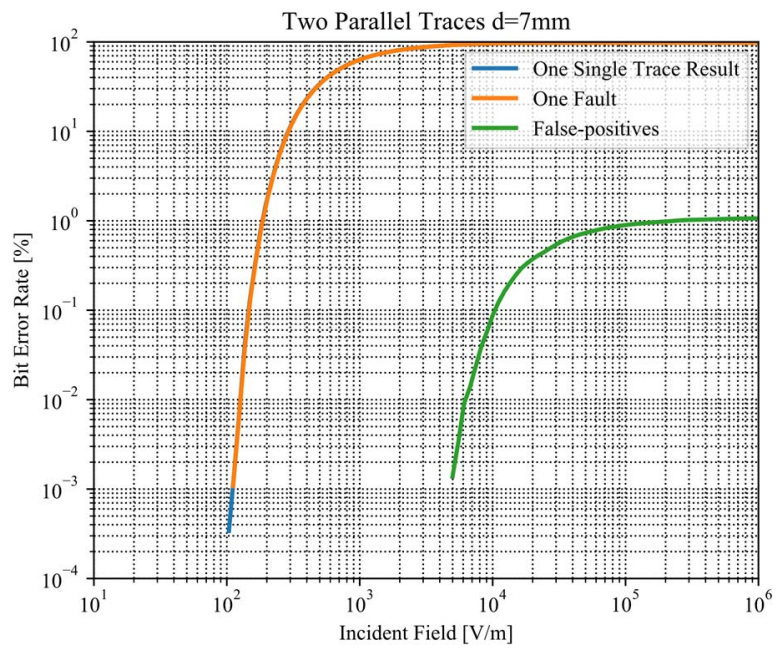

Fig. 6. BER for a single microstrip compared to BER for a 2-channel redundant system with inversion. The distance between the microstrips is $7 \mathrm{~mm}$, which causes a small amount of double faults (false-positives) to occur.

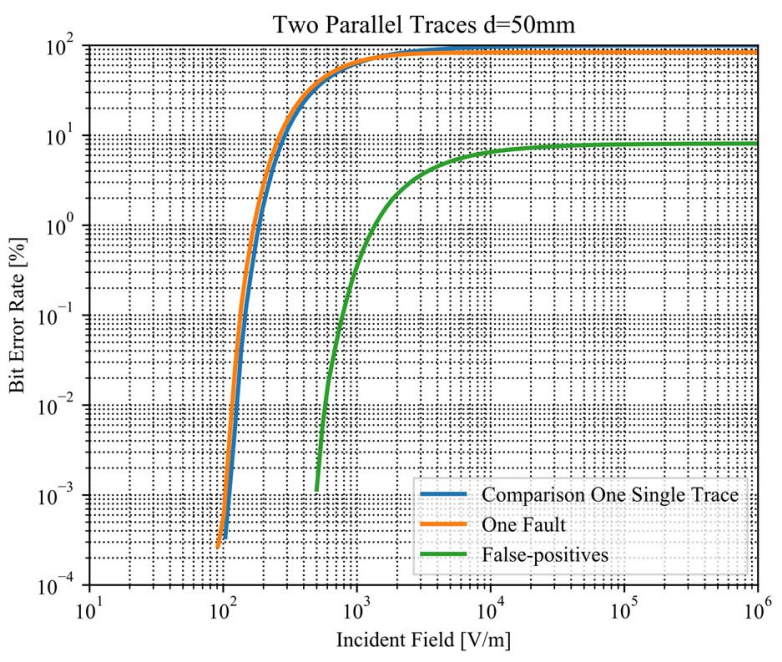

Fig. 7. BER for a single microstripe compared to BER for a 2-channel redundant system with inversion. The distance between the microstrips is 50 $\mathrm{mm}$, which causes much more double faults to occur.
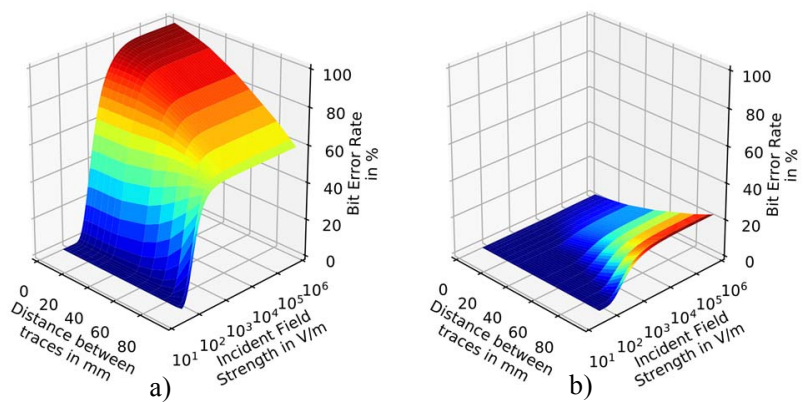

Fig. 8. Influence of the distance between the microstrips on the BER. In a) we see the single occurring faults, in b) we see the double occurring faults (false positives).

An interesting question is how the performance of a 2-channel redundant system compares to that of a differential pair (which, of course, doesn't comprise a comparator). The decoder of the differential pair takes the difference between the outputs, so that the disturbance is almost removed. Unless, once more, if there is a relatively large phase difference between the induced voltage on both microstrips. Figs. 9 and 10 show this comparison. It is seen that the differential pair starts to fail around the same level of incident field strength as the 2-channel redundant system. However, overall the 2-channel redundant channel behaves significantly better.

The fact that the BER for a differential pair goes to $50 \%$ for large incident field strength, can be explained as follows. Recall that we have a bit pattern with a $50 \%$ probability for a ' 0 ' and a $50 \%$ probability for a ' 1 ', then, for large disturbances:

$$
\begin{aligned}
& P(\text { Fault })=P(\text { Fault } \mid 0) P(0)+P(\text { Fault } \mid 1) P(1) \\
= & 0.5 * 0.5+0.5 * 0.5=0.25+0.25=0.5=50 \% .
\end{aligned}
$$




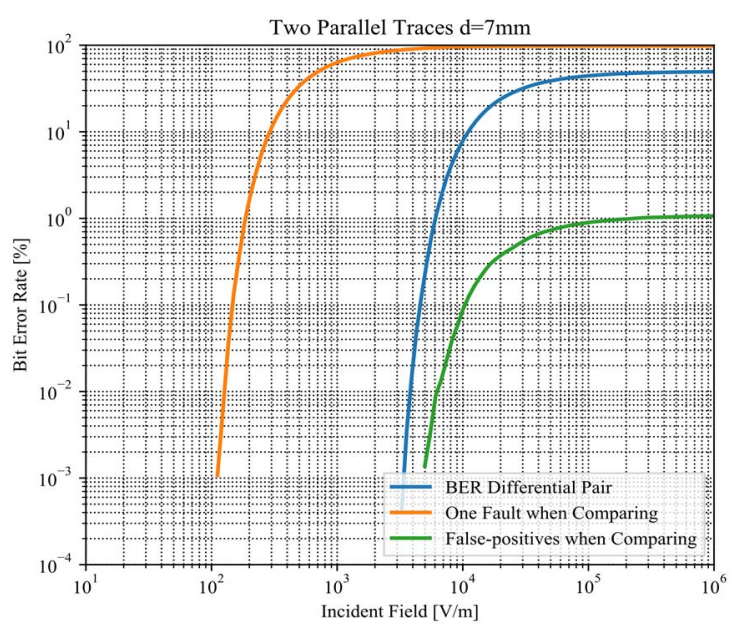

Fig. 9. Comparison between the BER for a differential pair and a 2-channel redundant system. The distance between the microstrips is $7 \mathrm{~mm}$.

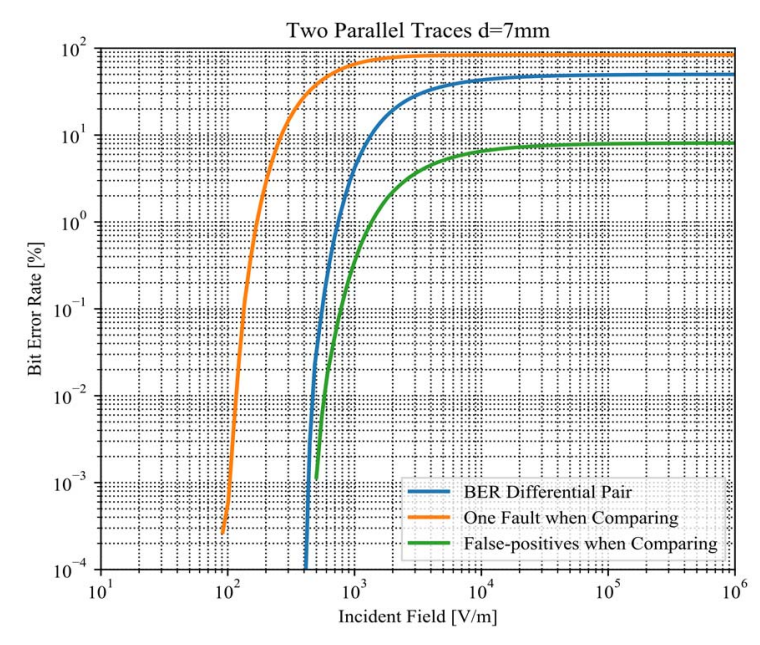

Fig. 10. Comparison between the BER for a differential pair and a 2-channel redundant system. The distance between the microstrips is $50 \mathrm{~mm}$.

\section{CONCLUSION}

This paper studied the effectiveness of inverse diversity to cope with interference caused by strong incident fields under reverberation room conditions. Two different geometries were compared: a non-redundant system comprising a single trace on a PCB and a redundant system comprising two parallel traces on a single PCB. A reciprocity-based technique was used to efficiently calculate the induced voltages and the resulting bit error probability in the different geometries. It is shown that the probability for false positive increases with increasing distance be- tween the two traces. For future work the combination of inversion and 2003 redundancy can be validated. Also creating a diverse fault tolerant system based on software techniques in combination with spatial diversity and inversion could be looked into.

\section{REFERENCES}

[1] http://eur-lex.europa.eu/legalcontent/NL/TXT/?uri=CELEX:32014L0030

[2] K. Armstrong, "Why EMC Immunity Testing is Inadequate for Functional Safety", Keith Armstrong, Proceedings of the IEEE International Symposium on EMC, Santa Clara, CA, August 9-13, pp. 145-149

[3] K. Armstrong. "Why increasing immunity test levels is not sufficient for high-reliability and critical equipment", Proceedings of the IEEE International Symposium on EMC 2009, Austin, TX, USA, pp. 30 - 35.

[4] D. Pissoort and K. Armstrong. "Why is the IEEE Developing a Standard on Managing Risks Due to EM Disturbances?" Proceedings of the IEEE International Symposium on EMC 2016, Ottawa, Canada, pp. $78-83$.

[5] F. Leferink, J. K. van der Ven, H. Bergsma and B. van Leersum, "Risk based EMC for complex systems," 2017 XXXIInd General Assembly and Scientific Symposium of the International Union of Radio Science (URSI GASS), Montreal, QC, 2017, pp. 1-4.

[6] The IET, "Code of Practice on Electromagnetic Resilience", 2017, www.theiet.org/resources/standards/emr-cop.cfm

[7] IEEE Standards Association, project P1848, "Techniques and Measures to Manage Functional Safety and Other Risks with Regard to Electromagnetic Disturbances", http://standards.ieee.org/

[8] D. Pissoort, J. Lannoo, J. Van Waes, A. Degraeve, J. Boydens, "Techniques and Measures to Achieve EMI Resilience in Mission- or Safety-Critical Systems," IEEE Electromagnetic Compatibility Magazine, vol. 6 quarter 4, 2017 [To Be Released]

[9] A. Degraeve and D. Pissoort, "Study of the effectiveness of spatially EMdiverse redundant systems under plane-wave illumination," 2016 AsiaPacific International Symposium on Electromagnetic Compatibility (APEMC), Shenzhen, 2016, pp. 211-213.

[10] A. Degraeve and D. Pissoort, "Study of the effectiveness of spatially EMdiverse redundant systems under reverberation room conditions, ” 2016 IEEE International Symposium on Electromagnetic Compatibility (EMC), Ottawa, ON, 2016, pp. 374-378.

[11] J. Lannoo, A. Degraeve, J. Boydens, D. Pissoort, "Study on the Use of Different Transmission Line Termination Strategies to Obtain EMI Diverse Redundant Systems," 2018 Joint IEEE International Symposium on Electromagnetic Compatibility (EMC) \& Asia-Pacific International Symposium on Electromagnetic Compatibility (APEMC), Singapore, 2018 [To Be Released]

[12] F. Vanhee, D. Pissoort, J. Catrysse, G. A. E. Vandenbosch and G. G. E. Gielen, "Efficient Reciprocity-Based Algorithm to Predict Worst Case Induced Disturbances on Multiconductor Transmission Lines due to Incoming Plane Waves," in IEEE Transactions on Electromagnetic Compatibility, vol. 55, no. 1, pp. 208-216, Feb. 2013.

[13] M. Magdowski, S. V. Tkachenko and R. Vick, "Coupling of Stochastic Electromagnetic Fields to a Transmission Line in a Reverberation Chamber," in IEEE Transactions on Electromagnetic Compatibility, vol. 53, no. 2, pp. 308-317, May 2011.

[14] D. A. Hill, "Plane wave in\&tegral representation for fields in reverberation chambers, " in IEEE Transactions on Electromagnetic Compatibility, vol. 40, no. 3, pp. 209-217, Aug 1998.

[15] Keysight Empro, Keysight Technologies, Nov. 2017. [Online]. Available: https://www.keysight.com/en/pc-1297143/empro-3d-em-simulationsoftware 